\title{
Improving Simulated Pediatric Airway Management in Community Emergency Departments Using a Collaborative Program With a Pediatric Academic Medical Center
}

\author{
Samer Abu-Sultaneh, Travis Whitfill, Courtney M Rowan, Matthew L Friedman, Kellie J Pearson, \\ Zachary J Berrens, Riad Lutfi, Marc A Auerbach, and Kamal Abulebda
}

\begin{abstract}
BACKGROUND: Pediatric airway management is a challenging process at community emergency departments (CEDs) due to lower pediatric volume, a lack of pediatric expertise among staff, and a lack of pediatric-specific equipment and resources. This has contributed to increased mortality in pediatric patients presenting to CEDs in comparison to pediatric academic medical centers (AMCs). We hypothesized that a collaborative program between CEDs and the state AMC would improve the quality of pediatric airway management provided by CEDs in simulated settings and the CEDs' pediatric emergency readiness scores. METHODS: This prospective, pre- and post-intervention study utilized in situ simulation and was conducted in 10 CEDs in the state of Indiana. A team from the pediatric AMC led a multi-faceted improvement program, which included post-simulation debriefing, addressing pediatric airway management issues, targeted assessment reports, access to pediatric resources, and ongoing communication with the AMC. The primary outcome of the study was improvement of simulated pediatric airway management in the CEDs. The secondary outcome was improvement of the CEDs' pediatric emergency readiness scores score. RESULTS: A total of 35 multidisciplinary teams participated in pre-intervention sessions, and 40 teams participated in post-intervention sessions. Overall adherence to a critical action checklist improved from $52 \%$ at the pre-intervention visits to $71 \%$ post-intervention $(P=.003)$. There were significant improvements in the use of appropriate endotracheal tube (ETT) size (from $67 \%$ to $100 \%, P=.02$ ), cuffed ETT (from $8 \%$ to $71 \%$, $P<.001$ ), appropriate blade size (from $58 \%$ to $100 \%, P=.03$ ), and availability of suction catheter (from $10 \%$ to $42 \%, P=.049$ ). The CEDs' total pediatric emergency readiness scores score improved from $58.8 \pm 15.6$ pre-intervention to $75.8 \pm 9.3$ post-intervention $(P=.01)$. CONCLUSIONS: A collaborative improvement program between a pediatric AMC and CEDs improved the CEDs' simulated pediatric emergency airway management. This model can be utilized to improve management of other pediatric critical conditions in these CEDs. Key words: high-fidelity simulation training; pediatric emergency medicine; airway management; intubation; interprofessional; multidisciplinary; community hospitals; community outreach. [Respir Care 2019;64(9):1073-1081. (c) 2019 Daedalus Enterprises]
\end{abstract}

Introduction

Pediatric airway emergencies are a challenging and anxiety-provoking task encountered in emergency depart-

\footnotetext{
Drs Abu-Sultaneh, Rowan, Friedman, Berrens, Lutfi, and Abulebda are affiliated with the Division of Pediatric Critical Care Medicine, Department of Pediatrics, Indiana University School of Medicine and Riley Hospital for Children at Indiana University Health, Indianapolis, Indiana. Dr Auerbach and Mr Whitfill are affiliated with the Department of Pediatrics and the Department of Emergency Medicine, Yale University School of Medicine,
}

ments. Each year, 34 million pediatric patient visits take place in $>5,000$ emergency departments in the United States, of which 4 million are high-acuity patients requiring emergent and lifesaving interventions. ${ }^{1}$ Critically ill

\footnotetext{
New Haven, Connecticut. Ms Pearson is affiliated with LifeLine Critical Care Transport, Indiana University Health, Indianapolis, Indiana.

Dr Abulebda presented a version of this paper at the International Meeting on Simulation in Healthcare 2018, held January 13-17, 2018, in Los Angeles, California.
} 
pediatric patients presenting to emergency departments require initial stabilization, which may include emergency airway management. ${ }^{2}$ These patients are often taken to the nearest location, which is most likely a community emergency department (CED). ${ }^{3-6}$ Current data show that $17 \%$ of pediatric patients require emergency airway management at CEDs prior to transport to an academic medical center (AMC). ${ }^{7}$

There are significantly more adverse events in pediatric airway management in community hospitals compared to tertiary pediatric emergency departments. ${ }^{8}$ In a large national study, pediatric subjects with respiratory arrest who were treated in teaching hospitals were found to have lower odds of mortality compared to such subjects in non-teaching hospitals. ${ }^{9}$ This is likely because a large proportion of pediatric patients are seen in low-volume CEDs that usually care for fewer than 15 pediatric patients a day. ${ }^{1}$ CEDs have limited access to pediatric-specific policies, procedures, and protocols, making them less ready to care for children..$^{2-5,10,11}$ Emergency physicians working in CEDs report being less comfortable performing lifesaving procedures in children, such as endotracheal intubation. ${ }^{12}$ This may be due to the challenges of maintaining the necessary skills for efficient and successful response to pediatric airway emergencies. Pediatric airway management carries a higher risk of intubation failure, airway compromise, and adverse events when compared to adults. ${ }^{13-15}$ Anatomical and physiological variances may lead to increased incidence of adverse events, such as intubation failure, hypoxemia, and right main stem intubation. ${ }^{16-18}$ When pediatric airway emergencies occur in CEDs, coordinated teamwork with immediate availability of pediatric-specific resources is critical for successful airway management.

The federal Emergency Medical Services for Children initiative implemented a number of programs aiming to improve pediatric readiness in CEDs. For example, the National Pediatric Readiness Project is a quality-improvement collaborative that aims to ensure that all CEDs have the essential resources to provide effective pediatric

\footnotetext{
The authors have disclosed financial support from Indiana University and from the Indiana University School of Medicine Department of Pediatrics. Mr Whitfill has disclosed a relationship with 410 Medical. The other authors have disclosed no conflicts of interest.

Supplementary material related to this paper is available at http:// www.rcjournal.com.

Correspondence: Samer Abu-Sultaneh MD, Department of Pediatrics, Division of Pediatric Critical Care, Indiana University School of Medicine and Riley Hospital for Children at Indiana University Health, 705 Riley Hospital Dr., Riley Phase 2 Room 4900, Indianapolis, Indiana 46202. E-mail: sultaneh@iu.edu.
}

DOI: $10.4187 /$ respcare .06750

\section{QUICK LOOK}

\section{Current knowledge}

Pediatric patients requiring airway management often present to community emergency departments for initial stabilization. Due to low pediatric volume in these centers, pediatric airway management in community emergency departments can be challenging.

\section{What this paper contributes to our knowledge}

We describe a collaborative initiative between a pediatric academic medical center and regional community emergency departments targeted at improving readiness for pediatric airway emergencies. Through in situ simulation, we demonstrated an improvement in pediatric airway management in the simulation setting.

care. ${ }^{19,20}$ Other pediatric emergency readiness initiatives highlight the importance of collaboration between CEDs and pediatric AMCs in using simulation to address quality of care in CEDs. ${ }^{21-24}$

A previous study demonstrated significant improvement in pediatric readiness scores in 10 CEDs after participation in a collaborative quality-improvement program. ${ }^{21}$ The study also identified deficiencies in the quality of care provided to critically ill children in the simulated setting. The deficiencies identified during this study were system gaps that had a strong potential to be associated with poor outcomes if performed in an actual clinical setting.

We hypothesized that a multi-faceted collaborative improvement program between the AMC and the CEDs could improve the quality of simulated pediatric airway management provided by multidisciplinary teams across a spectrum of CEDs in the state of Indiana.

\section{Methods}

\section{Study Design, Setting, and Population}

This was a prospective, pre- and post-interventional study of a selection of CEDs in the state of Indiana. The institutional review board at Indiana University School of Medicine approved this study. The study was conducted at 10 CEDs between May 2016 and August 2017. We selected 10 facilities from the 121 CEDs that deliver care for children based on their geographic location and historical patient transfer to the pediatric AMC. Team members from the pediatric AMC's critical care transport service served as program coordinators and educators. The CEDs' director or manager served as the pediatric champion for their 
CED. They were contacted to schedule the visits, and they recruited CED staff to participate in the simulation sessions. The project consisted of 3 phases: baseline, on-site assessments; targeted systemwide interventions; and postintervention improvement assessments.

\section{Baseline On-Site Assessment}

The baseline, on-site assessment consisted of the on-site pediatric emergency readiness score assessment and in situ simulations.

On-Site Pediatric Emergency Readiness Scores Assessment. The pediatric emergency readiness scores consists of 6 domains outlined in the National Pediatric Readiness Project: coordination of care, physician/nurse staffing, quality improvement, patient safety, policies/procedures, and equipment and supplies. ${ }^{3}$ During the baseline assessment visit, an in-person pediatric emergency readiness score was completed for each CED by 1 of 2 study personnel. These personnel were a registered nurse and respiratory therapist who underwent extensive training by the primary investigator $[\mathrm{KA}]$ and had $10 \mathrm{y}$ of pediatric critical care experience. More details about the methodology of on-site pediatric emergency readiness scores data collection are available in a previous publication. ${ }^{21}$

In Situ Simulation-Based Assessment. An AMC group of educators and experts in pediatric critical care medicine, pediatric emergency medicine, and pediatric critical care transport formed the Pediatric Community Outreach Mobile Education team. This team conducted baseline in situ simulation sessions. All team members were trained in simulation debriefing and completed a 2.5-d simulation instructor course at Indiana University School of Medicine prior to the study. ${ }^{25}$

CED participant teams were composed of 5-6 health care providers, including community emergency medicine physicians, nurses, respiratory therapists, and other health care providers (eg, physician assistants, pharmacists, and emergency medical services staff). Participants were protected from any clinical responsibilities during the simulations and debriefings. Each team participated in a 2.5-h in situ simulation session, which included a 6-month-old infant presenting to the CED with respiratory distress secondary to presumed viral bronchiolitis and progressing to respiratory failure requiring endotracheal intubation.

All sessions were conducted in the CEDs' resuscitation bay and used their own resources to enhance realism. Each session began with a standardized orientation including introduction to the team, mission of the project, and the day's agenda. Participants were oriented to the functionality of the high-fidelity simulators (SimBaby, Laerdal,
Stavanger, Norway). Laboratory data were provided on pre-printed laminated cards, available upon request, including standard point-of-care testing (ie, venous blood gas, dextrose, electrolytes) and a chest radiograph. These sessions assessed individual CED teams' performance and identified local CED systems issues and safety threats. After each simulation, the Pediatric Community Outreach Mobile Education team scored the CED team using a 16item simulation performance critical action checklist (see the supplementary materials at http://www.rcjournal.com).

\section{Targeted Systemwide Interventions}

The intervention phase consisted of post-simulation debriefing, pediatric-specific airway management issues, targeted assessment reports, and access to pediatric resources and ongoing communication with the AMC pediatric experts. Data from the simulation-based assessment and the pediatric emergency readiness scores results were used to guide interventions at each site and tailor the action plan accordingly.

Post-Simulation Debriefing. Following the in situ simulation, an educator-guided debriefing was conducted. During the debriefing, educators focused on engaging all CED team members to investigate individual and team performance, identify errors, and develop performance-improvement strategies via a reflective learning process. Educators used the traditional 3-phase structure model of "reaction, analysis, and summary" for the debriefing to maximize the learning experience among participants. ${ }^{26}$ Educators ensured that relevant issues and learning objectives were addressed during the debriefing. Participants were encouraged to explore any systems issues encountered during the simulated scenario. As an example, if the team used an inappropriate endotracheal tube (ETT) size, the educator would direct the team's attention through reflective questions such as "Was the appropriate tube size used?" and "If not, what needs to change to improve it?" This method allowed the team to identify their CED's system issues, such as a lack of appropriately sized ETTs.

Pediatric-Specific Airway Management Issues. Following the debriefing, a 15-20-min hands-on, bag-mask ventilation skills station was conducted to reinforce learning points encountered during the debriefing. CED teams were encouraged to acquire resources and equipment from their own CED. Participants were instructed on age-appropriate laryngoscope blade size, stylet, mask, and bag. Afterward, teams were guided through resources (eg, Breslow tape, smartphone applications, and Pediatric Advanced Life Support formulas) to locate the appropriate ETT size and depth. ETT cards with pre-calculated appropriate size/depth for different ages were distributed to par- 
ticipants (see the supplementary materials at http:// www.rcjournal.com). The benefits of using an appropriately sized cuffed over an uncuffed ETT for acutely ill children were discussed. The importance of using capnography during bag-mask ventilation and after intubation was highlighted.

Targeted Assessment Reports. A targeted assessment report including the baseline pediatric emergency readiness scores and the simulation-based performance was provided to the CED within 2 weeks of the initial in situ session. The assessment report included the missing items from each pediatric emergency readiness score domain and deviations from best practices as measure by the simulated performance. This report was presented in person to each CED's medical director or educator. During that meeting, a timeline of the next steps in the improvement process were discussed. In addition, CED-specific issues and concerns regarding the improvement process and any barriers were shared. An example of an assessment report with action items is available (see the supplementary materials at http://www.rcjournal.com).

Access to Pediatric Resources and Ongoing Communication. To ensure easy access to evidence-based best practices and pediatric resources, a website was created (http:// rileychildrens.org/pcome. Accessed March 14, 2019). This website included best practices, guidelines, algorithms, and high-quality educational modules focusing on the management of acute pediatric illnesses in the CEDs including respiratory failure. In addition, all CED sites were encouraged to contact the education team regarding any needed resources or additional assistance. As an example, when the assessment report identified that a site was missing a guideline required by the pediatric emergency readiness scores, the pediatric AMC would share a guideline and strategies for implementation in the community site.

\section{Post-Intervention Improvement Assessment}

Six months following the pediatric emergency readiness scores assessment and baseline simulation, the Pediatric Community Outreach Mobile Education team conducted a follow-up assessment of the same CED sites. An on-site pediatric emergency readiness score assessment was conducted using the same methodology used in the baseline visits. A follow-up in situ simulation session was performed at these CEDs using their actual clinical teams. All teams participated in a simulated case scenario of pediatric acute respiratory failure maintaining the same learning objectives with a different patient history (ie, patient age, weight, and initial presentation were changed, but the same scenario flow and objectives were used). The educator team, using the same critical action checklist as in the baseline assessment, scored the CED teams in the follow-up assessments. Constructive debriefing was performed after each session, and teams were given the opportunity to have hands-on practice and to utilize their CED's resources based on findings from the simulationbased assessment.

Outcome Measures. The main outcome was improvement in the CED's pediatric airway management as measured by the simulation critical action checklist. This critical action checklist was developed and adapted using best practice guidelines related to the management of pediatric respiratory failure. ${ }^{27-29}$ Content validity was provided through a consensus-based approach by a multidisciplinary expert panel composed of pediatric critical care, pediatric emergency medicine, and pediatric critical care transport providers and then adapted after being piloted within our institution. Operational definitions of the checklist are available (see the supplementary materials at http://www.rcjournal.com).

Performances were scored in real time independently by 2 educators based on the number of items performed correctly. Checklists were scored by assigning 1 point for each item performed correctly, and 0 points for each item not performed correctly. Scores were discussed between these educators until consensus was reached. Each CED team's performance score was calculated using equal weighting for all subcomponents and dividing by the total number of possible items to derive a score on a scale of $0-100$. The same educators ran the scenario and scored the checklist throughout the study period.

\section{Statistical Analyses}

Microsoft Excel version 14.0 (Microsoft, Redmond, Washington) was used for all data entry (simulation-based performance and pediatric readiness survey). All data were manually entered and transferred into SPSS version 22.0 (IBM, Armonk, New York) for statistical analysis. Data were examined for normality and homogeneity in each analysis. Improvement of the CEDs' assessments between pre- and post-intervention was examined using chi-square or Fisher exact tests for checklist items, independent $t$ tests for normal continuous data (i.e. team performance), and Wilcoxon-Mann Whitney $U$ tests for nonparametric data (i.e. pediatric readiness scores). Each CED was used as a unit regardless of number of participants. Mixed linear regression model was used to examine the impact of CED pediatric volume, presence of an in-patient pediatric unit, and the presence of a respiratory therapist in the team on team performance. 
Table 1. Characteristics of Participating Teams and Hospitals

\begin{tabular}{|c|c|c|}
\hline Variable & Pre-Intervention & Post-Intervention \\
\hline \multicolumn{3}{|l|}{ Team Characteristics } \\
\hline Number of teams, no. & 35 & 40 \\
\hline Number of participants, $n$ & 176 & 193 \\
\hline Physicians, $n(\%)$ & $35(19.9)$ & $39(20.2)$ \\
\hline Registered nurses, $n(\%)$ & $93(52.8)$ & $108(56.0)$ \\
\hline Respiratory therapists, $n(\%)$ & $15(8.5)$ & $18(9.3)$ \\
\hline Other, $n(\%)^{*}$ & $33(18.8)$ & $28(14.5)$ \\
\hline \multicolumn{3}{|l|}{ Hospital Characteristics } \\
\hline Number of hospitals, no. & 10 & 10 \\
\hline \multicolumn{3}{|l|}{ CED pediatric volume, no. $(\%)^{\dagger}$} \\
\hline Medium & $5(50)$ & $5(50)$ \\
\hline Medium-high & $5(50)$ & $5(50)$ \\
\hline Presence of in-patients pediatric units, no. (\%) & $7(70)$ & $7(70)$ \\
\hline Presence of PECC, no. (\%) & $7(70)$ & $10(100)$ \\
\hline \multicolumn{3}{|c|}{$\begin{array}{l}\text { * Included physician assistants, pharmacists and emergency medical services staff. } \\
\dagger \text { Medium }=1,800-4,999 \text { pediatric patients } / y \text {; medium-high }=5,000-9,999 \text { pediatric patients/y. } \\
\text { CED }=\text { community emergency department } \\
\text { PECC = pediatric emergency care coordinator }\end{array}$} \\
\hline
\end{tabular}

\section{Results}

\section{Participating CEDs and Team Characteristics}

Ten CEDs were enrolled in the study. A total of 35 multidisciplinary teams participated in pre-intervention assessment visits, and 40 teams participated in post-intervention assessment visits. Each CED had at least 3 teams participating in each of the pre- and post-intervention assessments. Twenty percent of the participants from pre-intervention assessment were present in the post-intervention assessments. Table 1 illustrates the demographics of the participating CEDs and provider teams.

\section{Pediatric Simulated Airway Management Outcomes}

Nine of the 10 CEDs demonstrated a significant improvement in overall pediatric airway management as measured with the critical action checklist, from 52\% preintervention to $71 \%$ post-intervention $(P=.003)$ (Fig. 1). Items that showed statistically significant improvement were the use of an age-appropriate ETT $(P=.02)$, use of a cuffed ETT $(P<.001)$, use of an appropriate laryngoscope blade $(P=.03)$, and availability of a suction catheter $(P=.049)$ (Table 2$)$. The rate of successful endotracheal intubation on first attempt was not significantly different between pre-intervention and post-intervention assessments (from $75 \%$ to $79 \%, P=.73$ ). Team performance was not affected by the presence of in-patient pediatric units in the CED, CED pediatric volume, or the presence of a respiratory therapist in the teams (Table 3).

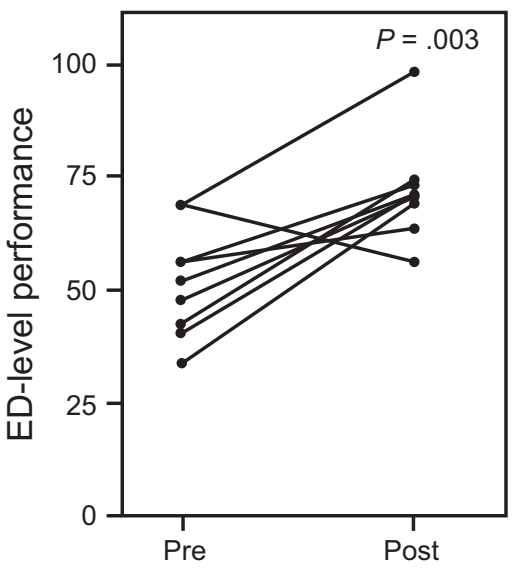

Fig. 1. Improvement in simulated airway management performance. Scores are indicated at the community emergency department (ED) level. $P$ value represents a 2 -sided independent $t$ test.

\section{Pediatric Emergency Readiness Scores}

The CEDs' total pediatric emergency readiness scores score (scaled from 0 to 100 ) improved from $58.8 \pm 15.6$ pre-intervention to $75.8 \pm 9.3$ post-intervention $(P=.01)$ (Table 4). All pediatric emergency readiness scores domains showed improvement, but only the following domains were statistically significant: patient safety improved by 4.2 points out of $14(P=.02)$, policies and procedures improved by 3.4 points out of $15.3(P=.03)$, and pediatric equipment improved by 1.8 points out of $34.7(P=.02)$. 
Table 2. Improvement of Team Performance During the Respiratory Failure Simulated Scenario

\begin{tabular}{|c|c|c|c|}
\hline Checklist Items & Pre-Intervention & Post-Intervention & $P$ \\
\hline 1. Appropriate ETT size used & $67(15-75)$ & $100(92-100)$ & .02 \\
\hline 2. Cuffed ETT used & $8(0-33)$ & $71(50-100)$ & $<.001$ \\
\hline 3. Cuffed checked (when cuffed ETT used) & $50(0-100)$ & $100(0-100)$ & .71 \\
\hline 4. Stylet used & $67(30-100)$ & $79(67-100)$ & .15 \\
\hline 5. Appropriate laryngoscope blade size used & $58(38-75)$ & $100(67-100)$ & .03 \\
\hline 6. Laryngoscope light checked & $82(67-100)$ & $100(83-100)$ & .67 \\
\hline 7. Suction catheter available & $10(0-33)$ & $42(19-63)$ & .049 \\
\hline 8. Bag and mask available & $100(96-100)$ & $100(96-100)$ & .85 \\
\hline 9. Time out performed & $0(0-0)$ & $0(0-8)$ & .18 \\
\hline 10. Patient's head positioned properly & $67(48-100)$ & $100(67-100)$ & .52 \\
\hline 11. Appropriate bagging technique & $58(33-88)$ & $79(46-100)$ & .051 \\
\hline 12. Laryngoscope blade inserted properly & $92(65-100)$ & $100(67-100)$ & .75 \\
\hline 13. ETT inserted to appropriate depth & $33(15-54)$ & $67(31-69)$ & .19 \\
\hline 14. Stylet removed (if used) & $100(100-100)$ & $100(100-100)$ & $>.99$ \\
\hline 15. ETT verified with $\mathrm{P}_{\mathrm{ETCO}}$ and chest auscultation & $58(30-71)$ & $92(54-100)$ & .16 \\
\hline 16. Chest radiograph ordered for confirmation & $67(58-100)$ & $79(67-100)$ & .52 \\
\hline Total adherence to checklist (out of 100) & $52 \pm 11.4$ & $70.9 \pm 10.9$ & .003 \\
\hline Intubation success rate & $75(65-100)$ & $79(67-100)$ & .73 \\
\hline
\end{tabular}

Data are expressed as the median (interquartile range) percentage of teams performing the checklist item correctly. There were 35 pre-intervention teams and 40 post-intervention teams. ETT $=$ endotracheal tube

$\mathrm{P}_{\mathrm{ETCO}}=$ end-tidal $\mathrm{CO}_{2}$ by capnography or colorimetry

Table 3. Teams' Performance by Hospital Characteristics

\begin{tabular}{|c|c|c|c|c|c|c|c|}
\hline \multirow[b]{2}{*}{ Variable } & \multirow[b]{2}{*}{ Overall } & \multicolumn{3}{|c|}{ In-Patient Pediatric Units } & \multicolumn{3}{|c|}{ CED Pediatric Volume* } \\
\hline & & $\begin{aligned} & \text { No } \\
\text { (no. }= & 3 \text { hospitals) }\end{aligned}$ & $\begin{array}{c}\text { Yes } \\
\text { (no. }=7 \text { hospitals) }\end{array}$ & $P$ & $\begin{array}{c}\text { Medium } \\
\text { (no. }=5 \text { hospitals) }\end{array}$ & $\begin{array}{l}\text { Medium-high } \\
\text { (no. }=5 \text { hospitals) }\end{array}$ & $P$ \\
\hline Team performance baseline & $52 \pm 11$ & $53 \pm 14$ & $52 \pm 12$ & .90 & $54 \pm 16$ & $51 \pm 7$ & .66 \\
\hline $\begin{array}{l}\text { Team performance improvement } \\
\text { from baseline }\end{array}$ & $19 \pm 15$ & $14 \pm 23$ & $21 \pm 11$ & .53 & $18 \pm 20$ & $19 \pm 8$ & .92 \\
\hline \multicolumn{8}{|c|}{$\begin{array}{l}\text { Data are expressed as mean } \pm \text { SD. } \\
* \text { Medium }=1,800-4,999 \text { pediatric patients/y; medium-high }=5,000-9,999 \text { pediatric patients } / y \text {. }\end{array}$} \\
\hline
\end{tabular}

\section{Discussion}

We describe an improvement initiative involving collaboration between a pediatric AMC and regional CEDs that led to improvement in pediatric emergency airway management measured during in situ simulations. Improvement was noted in almost all tasks of the critical action checklist using a customized, multi-faceted, systemwide intervention program. A significant improvement in pediatric emergency readiness scores was also noted in these CEDs.

This study utilized a novel approach involving in situ simulations as an investigative method to inform the AMC development of targeted improvement interventions for the participating CEDs. These results are consistent with prior work that demonstrated improved pediatric emer- gency preparedness in programs involving partnerships between AMCs and CEDs. ${ }^{21,23,30,31}$

Simulation-based interventions in emergency settings traditionally involve the training of health care providers and teams for high-stake, low-frequency events. ${ }^{32,33}$ Studies have reported on simulations' utility as a tool for skills and knowledge acquisition in pediatric acutecare providers. ${ }^{34-36}$ More specifically, simulation-based outreach education by AMCs to CEDs has been reported to improve the management of simulated pediatric traumas prior to transfer to an AMC. ${ }^{37,38}$ While these studies have reported that simulation improves individual and team performance in acute care setting, improved outcomes in the simulated setting do not guarantee the translation of these improvements into actual clinical care. ${ }^{39}$ 
Table 4. Pre- and Post-Intervention Pediatric Readiness Score

\begin{tabular}{|c|c|c|c|}
\hline Variable & Pre-Intervention (no. $=10$ hospitals) & Post-Intervention (no. $=10$ hospitals) & $P$ \\
\hline Total Pediatric Readiness Score (max of 100 ) & $58.8 \pm 15.6$ & $75.8 \pm 9.3$ & .01 \\
\hline Coordination of pediatric patient care (max of 19 ) & $9.5(2.4-9.5)$ & $14.3(9.5-19)$ & .059 \\
\hline Staffing median (max of 10$)$ & $5.0(1.3-5.0)$ & $5.0(5.0-5.0)$ & .18 \\
\hline Quality improvement (max of 7) & $0(0-3.8)$ & $3.3(0-6.5)$ & .11 \\
\hline Patient safety (max of 14 ) & $9.8(9.1-12.1)$ & $14.0(11.4-14.0)$ & .02 \\
\hline Policies and procedures (max of 15.3 ) & $6.9(4.2-10.6)$ & $10.3(6.5-11.5)$ & .03 \\
\hline Pediatric equipment ( $\max$ of 34.7 ) & $28.0(26.0-29.8)$ & $29.8(28.3-31.8)$ & .02 \\
\hline
\end{tabular}

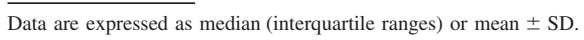

Our study has 2 important differences from previous research. First, we had success in using simulation as a quality-improvement modality to evaluate the processes of care and target systems issues in CEDs. This program used simulation to promote systemwide evaluation and improvement, rather than focusing on individual team member performance.

Second, the in situ simulation allowed CED teams to apply their knowledge, using their own equipment and guidelines, in their actual clinical settings. This created a framework to provide specific and targeted information on gaps in the delivery of care in each participating CED. Debriefing provided opportunities to explore teams' performances and highlight gaps encountered during the simulated session. This included the participants' identification of local system-related issues in each CED. For example, when an in situ simulation revealed the lack of age-appropriate ETTs and capnographs, the post-simulation debriefing emphasized the need for this equipment to deliver optimal care. This provided a strong argument to the CED leadership to ensure the availability of this equipment, which in turn improved the equipment and supplies domain of pediatric emergency readiness scores. As another example, many teams identified the need to transfer the patient to the tertiary pediatric center, but it was noted that many of the CEDs did not have transfer guidelines readily available. This triggered a process to obtain transfer guidelines, which led to significant improvement in the post-intervention policies and procedures pediatric emergency readiness scores domain.

The targeted assessment report was a unique component of the study. This report included an overview of team performance, deficiencies noted during the simulations, and the pediatric emergency readiness scores. In addition, it included a detailed action plan with a timeline for improvement. This document was a supportive tool for pediatric emergency care champions to engage CED leadership in addressing local issues and implementing systems changes. Furthermore, distribution of the educational materials (ie, laminated cards) and clinical pathways allowed all CED providers to have resources available for pediatric airway management, not just those involved in the intervention. The use of clinical pathways and best practice guidelines has enhanced patient safety and improved pediatric patients' outcomes in emergency departments..$^{40,41}$ We found this in our study as well, because the majority of CED sites used our ETT cards during the follow-up assessment simulation visits (see the supplementary materials at http://www.rcjournal.com). There was also a significant increase in the use of cuffed ETTs at the follow-up assessment visits, which is preferred when intubating children with respiratory failure. ${ }^{28,42}$ In addition, our educational website was accessed $>500$ times during the study period.

Throughout the study period, all CED pediatric champions maintained ongoing communication with the educator team, which had a positive impact on addressing CEDs' educational needs and potential barriers in the improvement process. As an example, when a certain guideline or policy regarding pediatric care was missing, the CED champion could reach out to the team at the AMC to learn about guidelines and strategies for implementation available at the pediatric AMC. Similarly, if the CED was noted to have gaps in the simulation-based performance, the educator team could provide the CED with resources for training or consultation.

Overall, the goal of our study was to determine the impact of our simulation-based collaborative program in promoting CED system-level changes and strategies to enable sustained improvement by the entire CED system, rather than the traditional use of simulation in improving individual performance or educational outcomes only. This is reflected in the finding that $80 \%$ of providers in the post-intervention sessions were not the same providers who participated in the pre-intervention sessions, yet there was an overall improvement in pediatric airway management and marked improvement in 4 action items in the critical action checklist (ie, use of age-appropriate ETT, use of cuffed ETT, use of appropriate laryngoscope blade size, and availability of suction catheter). The improved utilization can be attributed to the systems-level impact of the distribution of ETT cards to staff. The improved availabil- 
ity of equipment and supplies can be attributed to the assessment reports.

Our findings are consistent with other reports of simu-

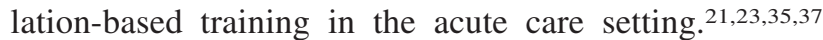
Katznelson et $\mathrm{al}^{30}$ reported that longitudinal in situ outreach simulation improved community providers' comfort and performance in a simulated setting. Hebbar et $\mathrm{al}^{41}$ found improved quality of patient care hospitalwide through integration of a simulation-based training program, which led to better adherence to best practices and decreased error rates. Andreatta et $\mathrm{al}^{43}$ demonstrated that a simulation-based program improved pediatric cardiopulmonary arrest survival rates and outcomes at a major children's hospital. These reports are particularly important because they relate the translational outcomes of the simulationbased improvement programs, beginning in the simulation environment, driving improved patient care practices, and ending with better patient outcomes. Interestingly, CEDs' pediatric volume or the presence of an in-patient pediatric unit did not affect teams' performance in our study. Although this is hard to interpret accurately due to the small sample size, other studies have stressed the need to target intervention in CEDs with lower pediatric volumes. ${ }^{1-3,5}$

The impact of this program highlights the importance of collaboration between AMCs and regional CEDs. The described program can be replicated in other regions or states as an effective strategy to improve the processes of care and potentially improve patient outcomes in hospitals that care for fewer children. Future studies must explore the cost-benefit of this type of program to provide funding outside of a research project, as well as the impact of this work on patient outcomes. In addition, this collaborative model can be considered for other populations and conditions.

Our study has some limitations. First, only $10 \%$ of CEDs in the state were included in the study, so the results may not be generalizable to other CEDs. However, the CEDs included had a mix of medium and medium high pediatric volumes at varying distances from the AMC. Second, the time to intubate and number of attempts to successful intubation were not studied because the main aim of the study was to assess and improve overall team preparedness for pediatric airway management rather than the procedural skill of intubation itself. The third limitation is that the study outcome measures did not include actual patient care processes or outcomes. However, by emphasizing adherence to best practices and evidence-based medicine guidelines as well as enhancing CED preparedness, we anticipate that this ultimately leads to improved patient care.

\section{Conclusions}

A collaborative program between a pediatric AMC and CEDs was successful in improving the CEDs' simulated pediatric emergency airway management and pediatric readiness scores. The use of in situ simulation, combined with the partnership between the pediatric AMC and CEDs, was successful in potentiating system-level changes in the CEDs. Such a collaborative model may guide national efforts to improve the process of care, and ultimately patient outcomes, in the acute care setting.

\section{ACKNOWLEDGMENTS}

The authors thank the pediatric critical care team at Riley Hospital for Children at Indiana University Health and Indiana University Health Lifeline team for their support and help in this project. The authors also acknowledge the contributions of members of the International Network for Simulation-Based Pediatric Innovation, Research, and Education (INSPIRE), which helped shape this project.

\section{REFERENCES}

1. Whitfill T, Auerbach M, Scherzer DJ, Shi J, Xiang H, Stanley RM. Emergency care for children in the United States: epidemiology and trends over time. J Emerg Med 2018;55(3):423-434.

2. Warren J, Fromm RE Jr, Orr RA, Rotello LC, Horst HM, American College of Critical Care Medicine. Guidelines for the inter- and intrahospital transport of critically ill patients. Crit Care Med 2004; 32(1):256-262.

3. Gausche-Hill M, Ely M, Schmuhl P, Telford R, Remick KE, Edgerton EA, et al. A national assessment of pediatric readiness of emergency departments. JAMA Pediatr 2015;169(6):527-534.

4. Auerbach M, Whitfill T, Gawel M, Kessler D, Walsh B, Gangadharan $\mathrm{S}$, et al. Differences in the quality of pediatric resuscitative care across a spectrum of emergency departments. JAMA Pediatr 2016;170(10):987-994.

5. Kessler DO, Walsh B, Whitfill T, Dudas RA, Gangadharan S, Gawel $\mathrm{M}$, et al. Disparities in adherence to pediatric sepsis guidelines across a spectrum of emergency departments: a multicenter, cross-sectional observational in situ simulation study. J Emerg Med 2016;50(3):403415.e1-3.

6. Gausche-Hill M, Schmitz C, Lewis RJ. Pediatric preparedness of US emergency departments: a 2003 survey. Pediatrics 2007;120(6):12291237.

7. Nishisaki A, Marwaha N, Kasinathan V, Brust P, Brown CA 3rd, Berg RA, et al. Airway management in pediatric patients at referring hospitals compared to a receiving tertiary pediatric ICU. Resuscitation 2011;82(4):386-390.

8. Easley RB, Segeleon JE, Haun SE, Tobias JD. Prospective study of airway management of children requiring endotracheal intubation before admission to a pediatric intensive care unit. Crit Care Med 2000;28(6):2058-2063.

9. Hansen M, Fleischman R, Meckler G, Newgard CD. The association between hospital type and mortality among critically ill children in US EDs. Resuscitation 2013;84(4):488-491.

10. Ray KN, Olson LM, Edgerton EA, Ely M, Gausche-Hill M, Schmuhl $\mathrm{P}$, et al. access to high pediatric-readiness emergency care in the United States. J Pediatr 2018;194:225-232.

11. Neuman MI, Shah SS, Shapiro DJ, Hersh AL. Emergency department management of childhood pneumonia in the United States prior to publication of national guidelines. Acad Emerg Med 2013;20(3): 240-246.

12. Simon HK, Sullivan F. Confidence in performance of pediatric emergency medicine procedures by community emergency practitioners. Pediatr Emerg Care 1996;12(5):336-339. 


\section{Improving Simulated Pediatric Airway Management}

13. Stinson HR, Srinivasan V, Topjian AA, Sutton RM, Nadkarni VM, Berg RA, et al. Failure of invasive airway placement on the first attempt is associated with progression to cardiac arrest in pediatric acute respiratory compromise. Pediatr Crit Care Med 2018;19(1): 9-16.

14. Kerrey BT, Rinderknecht AS, Geis GL, Nigrovic LE, Mittiga MR. Rapid sequence intubation for pediatric emergency patients: higher frequency of failed attempts and adverse effects found by video review. Ann Emerg Med 2012;60(3):251-259.

15. Shiima Y, Berg RA, Bogner HR, Morales KH, Nadkarni VM, Nishisaki A, et al. Cardiac arrests associated with tracheal intubations in PICUs: A multicenter cohort study. Crit Care Med 2016;44(9):16751682.

16. Graciano AL, Tamburro R, Thompson AE, Fiadjoe J, Nadkarni VM, Nishisaki A. Incidence and associated factors of difficult tracheal intubations in pediatric ICUs: a report from National Emergency Airway Registry for Children: NEAR4KIDS. Intensive Care Med 2014;40(11):1659-1669.

17. Lee JH, Turner DA, Kamat P, Nett S, Shults J, Nadkarni VM, et al. The number of tracheal intubation attempts matters! A prospective multi-institutional pediatric observational study. BMC Pediatr 2016; 16:58.

18. Luten RC, Mick NW. Differentiating aspects of the pediatric airway. In: Walls RM, Murphy MF, editors. Manual of Emergency Airway Management. Philadelphia: Wolters Kluwer/Lippincott Williams \& Wilkins Health; 2012:275-292.

19. Sadovich J, Adirim T, Telford R, Olson LM, Gausche-Hill M, Edgerton EA. Pediatric readiness in Indian health service and tribal emergency departments: results from the National Pediatric Readiness Project. J Emerg Nurs 2017;43(1):49-56.

20. Remick K, Kaji AH, Olson L, Ely M, Schmuhl P, McGrath N, et al. Pediatric readiness and facility verification. Ann Emerg Med 2016; 67(3):320-328.

21. Abulebda K, Lutfi R, Whitfill T, Abu-Sultaneh S, Leeper KJ, Weinstein E, et al. A collaborative in situ simulation-based pediatric readiness improvement program for community emergency departments. Acad Emerg Med 2018;25(2):177-185.

22. Rosen JR, Suresh S, Saladino RA. Quality care and patient safety in the pediatric emergency department. Pediatr Clin North Am 2016; 63(2):269-282.

23. Whitfill T, Gawel M, Auerbach M. A simulation-based quality improvement initiative improves pediatric readiness in community hospitals. Pediatr Emerg Care 2018;34(6):431-435.

24. Kelley-Quon LI, Crowley MA, Applebaum H, Cummings K, Kang $\mathrm{RJ}$, Tseng $\mathrm{CH}$, et al. Academic-community partnerships improve outcomes in pediatric trauma care. J Pediatr Surg 2015;50(6):10321036.

25. Byrnes BJ. Simulation instructor course. Available at: https://medicine. iu.edu/departments/pediatrics/specialties/neonatal-perinatal/education/ simulation-instructor-course/. Accessed Oct 1, 2018

26. Zigmont JJ, Kappus LJ, Sudikoff SN. The 3D model of debriefing: defusing, discovering, and deepening. Semin Perinatol 2011;35(2): 52-58.

27. Hazinski MF, Zaritsky AL, Chameides L, Pedersen AJ, Adirim T, American Heart Association, et al. Airway, ventilation and management of respiratory distress and failure. In: Pediatric Advanced Life Support (Pals) Provider Manual. Dallas: American Heart Association; 2002:81-126.

28. Kleinman ME, Chameides L, Schexnayder SM, Samson RA, Hazinski MF, Atkins DL, et al. Part 14: pediatric advanced life support:
2010 American Heart Association guidelines for cardiopulmonary resuscitation and emergency cardiovascular care. Circulation 2010; 122(18 Suppl 3):S876-908.

29. Nishisaki A, Donoghue AJ, Colborn S, Watson C, Meyer A, Niles D, et al. Development of an instrument for a primary airway provider's performance with an ICU multidisciplinary team in pediatric respiratory failure using simulation. Respir Care 2012;57(7):1121-1128.

30. Katznelson JH, Wang J, Stevens MW, Mills WA. Improving pediatric preparedness in critical access hospital emergency departments: impact of a longitudinal in situ simulation program. Pediatr Emerg Care 2018;34(1):17-20.

31. Walls TA, Hughes NT, Mullan PC, Chamberlain JM, Brown K. Improving pediatric asthma outcomes in a community emergency department. Pediatrics 2017;139(1):e20160088.

32. Falcone RA Jr, Daugherty M, Schweer L, Patterson M, Brown RL, Garcia VF. Multidisciplinary pediatric trauma team training using high-fidelity trauma simulation. J Pediatr Surg 2008;43(6):1065-1071.

33. Bond WF, Lammers RL, Spillane LL, Smith-Coggins R, Fernandez $\mathrm{R}$, Reznek MA, et al. The use of simulation in emergency medicine: a research agenda. Acad Emerg Med 2007;14(4):353-363.

34. Hunt EA, Walker AR, Shaffner DH, Miller MR, Pronovost PJ. Simulation of in-hospital pediatric medical emergencies and cardiopulmonary arrests: highlighting the importance of the first 5 minutes. Pediatrics 2008;121(1):e34-e43.

35. Allan CK, Thiagarajan RR, Beke D, Imprescia A, Kappus LJ, Garden A, et al. Simulation-based training delivered directly to the pediatric cardiac intensive care unit engenders preparedness, comfort, and decreased anxiety among multidisciplinary resuscitation teams. J Thorac Cardiovasc Surg 2010;140(3):646-652.

36. Weinberg ER, Auerbach MA, Shah NB. The use of simulation for pediatric training and assessment. Curr Opin Pediatr 2009;21(3): 282-287.

37. Bayouth L, Ashley S, Brady J, Lake B, Keeter M, Schiller D, et al. An in-situ simulation-based educational outreach project for pediatric trauma care in a rural trauma system. J Pediatr Surg 2018;53(2): 367-371.

38. Hunt EA, Heine M, Hohenhaus SM, Luo X, Frush KS. Simulated pediatric trauma team management: assessment of an educational intervention. Pediatr Emerg Care 2007;23(11):796-804.

39. Andreatta PB, Woodrum DT, Birkmeyer JD, Yellamanchilli RK, Doherty GM, Gauger PG, et al. Laparoscopic skills are improved with LapMentor training: results of a randomized, double-blinded study. Ann Surg 2006;243(6):854-860.

40. Chin R, Browne GJ, Lam LT, McCaskill ME, Fasher B, Hort J. Effectiveness of a croup clinical pathway in the management of children with croup presenting to an emergency department. J Paediatr Child Health 2002;38(4):382-387.

41. Hebbar KB, Colman N, Williams L, Pina J, Davis L, Bost JE, et al. A quality initiative: a systemwide reduction in serious medication events through targeted simulation training. Simul Healthc 2018; 13(5):324-330

42. Chen L, Zhang J, Pan G, Li X, Shi T, He W. Cuffed versus uncuffed endotracheal tubes in pediatrics: a meta-analysis. Open Med (Wars) 2018;13:366-373

43. Andreatta P, Saxton E, Thompson M, Annich G. Simulation-based mock codes significantly correlate with improved pediatric patient cardiopulmonary arrest survival rates. Pediatr Crit Care Med 2011; 12(1):33-38. 